University of Wollongong

Research Online

Faculty of Engineering and Information

Faculty of Engineering and Information

Sciences - Papers: Part A

Sciences

$1-1-2020$

\title{
Model-free adaptive iterative learning control of melt pool width in wire arc additive manufacturing
}

Chunyang Xia

University of Wollongong, cx772@uowmail.edu.au

Zengxi Stephen Pan

University of Wollongong, zengxi@uow.edu.au

Shiyu Zhang

University of Wollongong, sz946@uowmail.edu.au

Hui Jun Li

University of Wollongong, huijun@uow.edu.au

Yanling $\mathrm{Xu}$

See next page for additional authors

Follow this and additional works at: https://ro.uow.edu.au/eispapers

Part of the Engineering Commons, and the Science and Technology Studies Commons

Research Online is the open access institutional repository for the University of Wollongong. For further information contact the UOW Library: research-pubs@uow.edu.au 


\title{
Model-free adaptive iterative learning control of melt pool width in wire arc additive manufacturing
}

\author{
Abstract \\ (C) 2020, Springer-Verlag London Ltd., part of Springer Nature. Wire arc additive manufacturing (WAAM) is \\ a Direct Energy Deposition (DED) technology, which utilize electrical arc as heat source to deposit metal \\ material bead by bead to make up the final component. However, issues like the lack of assurance in \\ accuracy, repeatability and stability hinder the further application in industry. Therefore, a Model Free \\ Adaptive Iterative Learning Control (MFAILC) algorithm was developed to be applied in WAAM process in \\ this study. The dynamic process of WAAM is modelled by adaptive neuro fuzzy inference system (ANFIS). \\ Based on this ANFIS model, simulations are performed to demonstrate the effectiveness of MFAILC \\ algorithm. Furthermore, experiments are conducted to investigate the tracking performance and \\ robustness of the MFAILC controller. This work will help to improve the forming accuracy and automatic \\ level of WAAM. \\ Disciplines \\ Engineering | Science and Technology Studies

\section{Publication Details} \\ Xia, C., Pan, Z., Zhang, S., Li, H., Xu, Y. \& Chen, S. (2020). Model-free adaptive iterative learning control of \\ melt pool width in wire arc additive manufacturing. International Journal of Advanced Manufacturing \\ Technology, 110 (7-8), 2131-2142. \\ Authors \\ Chunyang Xia, Zengxi Stephen Pan, Shiyu Zhang, Hui Jun Li, Yanling Xu, and Shanben Chen
}




\title{
Model free adaptive iterative learning control of melt pool width in wire arc additive manufacturing
}

\author{
Chunyang Xia ${ }^{\mathrm{a}, \mathrm{b}}$, Zengxi Pan ${ }^{\mathrm{a}, *}$, Shiyu Zhang ${ }^{\mathrm{a}}$, Huijun $\mathrm{Li}^{\mathrm{a}}$, Yanling Xu${ }^{\mathrm{b}}$, Shanben $\mathrm{Chen}^{\mathrm{b}}$ \\ ${ }^{\text {a }}$ School of Mechanical, Materials, Mechatronic and Biomedical Engineering, University of \\ Wollongong, Wollongong, NSW, 2522, Australia \\ ${ }^{\mathrm{b}}$ School of Materials Science and Engineering, Shanghai Jiao Tong University, Shanghai \\ 200240, China
}

Corresponding author: Zengxi Pan

Email: zengxi@uow.edu.au

Postal address: Northfields Ave Wollongong, NSW 2522 Australia

Telephone numbers: +61242215498

\begin{abstract}
Wire arc additive manufacturing (WAAM) is a Direct Energy Deposition (DED) technology, which utilize electrical arc as heat source to deposit metal material bead by bead to make up the final component. However, issues like the lack of assurance in accuracy, repeatability and stability, hinder the further application in industry. Therefore, a Model Free Adaptive Iterative Learning Control (MFAILC) algorithm was developed to be applied in WAAM process in this study. The dynamic process of WAAM is modelled by adaptive neuro fuzzy inference system (ANFIS). Based on this ANFIS model, simulations are performed to demonstrate the effectiveness of MFAILC algorithm. Furthermore, experiments are conducted to investigate the tracking performance and robustness of the MFAILC controller. This work will help to improve the forming accuracy and automatic level of WAAM.
\end{abstract}

Keywords: model free adaptive; Iterative learning control; additive manufacturing; ANFIS; WAAM

\section{Introduction}

Over the past decades, additive manufacturing (AM) technology has been developed continuously due to its advantages in design flexibility and time efficiency. Considered as a game changing technology [1], AM technology has been applied in many fields, such as aerospace [2], automotive [3], biomedical [4] [5] and architectural design. Nowdays, AM techniques has been extend to rapid repair and rapid tooling from original direct fabrication[6]. 
As a result, AM industry grows rapidly and its value estimated to be over $\$ 30$ billion by 2022 [7].

Wire arc additive manufacturing (WAAM) is a new emerging AM technology, which makes use of an electric arc as heat-source to deposit metal material layer-by-layer, which makes up the final part (as illustrated in Figure 1). It is one of fast growing direct forming technology in AM field in recent years. Compare to other AM method, it possess the features of higher deposition efficiency, lower equipment cost, higher material utilization rate and clear production environment [8]. However, as pointed out in related literatures[9] [10], precision and repeatability during AM process hind its further development. To overcome those challenges, in-situ monitoring and control have received increasing attention, which were considered as an effective way to improve the final quality of production. According to a road map workshop on measurement science needs for metal-based AM [26], process monitoring and feedback control for AM process were identified as key advancements critical to the overall methods' success.

In traditional arc welding field, there are already some profound studies on process sensing and control. For example, Zhang et al [11-13] using active vision method to measure the three dimension information of melt pool, and implement feedback control based on model predictive control algorithm. Xiong et al [14] developed a visual sensing based fuzzy controller to control the penetration during MIG welding process. Although arc welding and WAAM share the utilization of an electric arc as the heat source, the objectives for control issues are not quite the same. Welding control emphasizes the penetration while WAAM should address the control of layer geometry.

As a new emerging technology, the studies on process control for WAAM is still fewer. In early study, Doumanidis et al $[15,16]$ developed a multi-variable adaptive controller for the WAAM process, which is based on generalized one step ahead control algorithm. Xiong [17] et al proposed to use a single neuron self-leaning controller to regulate the bead width during WAAM. Besides, a Model identification adaptive controller (MIAC) was developed by Xiong [18] to control the bead height during the WAAM process. To maintain the deposited height, Li et al. [19] proposed an adaptive process control scheme (APCS), which divided the too-path into several segments according to the shape corner distribution, and provided different travel speed for each segment according to a process model. To maintain the process stability of WAAM, Reisgen et al. [20] implemented torch height and workpiece height control based on image processing. Radel et al. [21] implemented closed-loop control for skeleton WAAM. A camera was utilized to detect the contour of the deposit and computes its current position, and the future deposition can be corrected by CAM software. Recently, Xiong et al. [22] proposed to combine the height information of both previous and current layers in PID based closed-loop control, which could help increase the response speed of the control system.

WAAM involves complex physical process, and process variables couple with each other. It's hard to obtain accurate mathematical model for WAAM. Therefore, model-free control algorithms are call for to handle such complicated systems with uncertain mathematical models and highly nonlinear task requirements. Iterative Learning Control (ILC) is an intelligence control strategies [19], which was proposed to deal with the control task in repetitive process, such as robotic manipulators and chemical batch processes. The basic idea of ILC is to learn from the previous experience to improve future control performance. In traditional ICL 
algorithm, like P-ICL, PD-ILC and PID-ILC, the learning gains are fixed. During iterative learning process, the calculation of control input only rely on feedback signal, and the mapping relationship and learning gains are kept constant. But in practice, controlled system may be time-variant and the initial condition is hard to be maintained. Fixed learning gain algorithm may be not capable to achieve fast tracking effect. Hou [20] [21] proposed Model-Free Adaptive Control for nonlinear systems in his doctoral thesis. A new concept of pseudo-partial derivative (PPD) was proposed to achieve dynamic linearization. An equivalent linearized data model was built at each working instant, and PPD was updated online based on system's I/O data. Chi et al. [22] proposed a Model Free Adaptive Iterative Learning Control algorithm, which combined both adaptability and learning ability from Iterative Learning Control and Model Free Adaptive Control. The learning gain of ILC could be updated online based on the estimated value of PPD. This makes it more effective for controlling process, which is difficult modelling, strong nonlinear and time varying. The implement of MFAILC don't require any information of dynamic model, and the limitation of model uncertainties can be overcome. MFAILC has been attracting attention from both academia and industry. Bu et al. [27] applied MFAILC for farm vehicle path tracking. It's found that this algorithm could compensate for the effect of initial shifting to preserve perfect tracking in vehicle path tracking. Zhang, Hu [28] applied MFAICL for a pneumatic muscle-driven robot, and progressive tracking of the desired track of the pneumatic muscle was achieved. Cao et al. [29] proposed to utilize MFAICL to control the tool feed system in noncircular turning.

In the WAAM deposition process, a controllable melt pool width could help improve the manufacturing accuracy. The tool path of WAAM is pre-set before deposition, which demands the bead geometry controllable. Also, for some complex components, a varying deposition bead width may be required. Besides, with the number of deposition layers increasing, the thermal boundary may shift, constant parameters can't maintain the desired bead width. Therefore, it's necessary to control the melt pool width during the WAAM process. WAAM involves complex physical process, and it's difficult to establish an accurate dynamic model. Also, the layer-bylayer deposition process of WAAM can be viewed as a repetitive mode. Due to these characteristics, iteratively learning control is extremely appropriate for the WAAM process. Therefore, this study proposes to apply MFAILC algorithm to control the melt pool width during WAAM, which only requires the I/O data.

The article will be organized as follows: in section 2, the dynamic model of WAAM for simulation will be established. Section 3 derive the MAFILC algorithm for WAAM. In section 4 , simulation is conducted to investigate the effectiveness of MAFILC. To further validate the algorithm, experiments are designed and implemented in section 5. Section 6 concludes this article. 


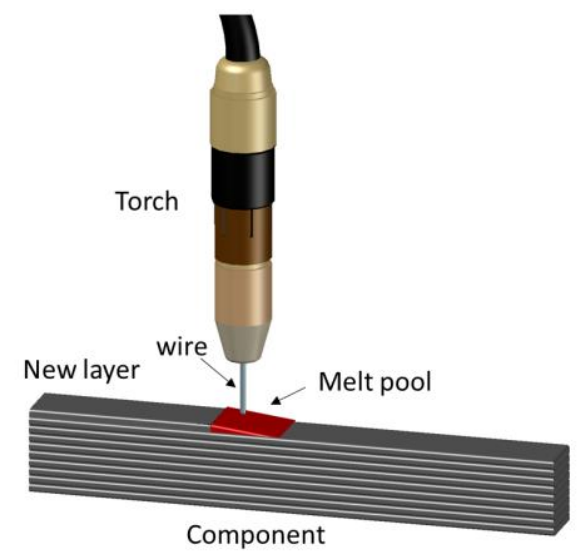

Figure 1 Diagram of WAAM process

\section{ANFIS based modelling}

The melting and solidification process of metal wire in WAAM involves complex physical phenomenon process, so it's hard to derive the dynamic model of WAAM in the form of mechanism. Therefore, a data-driven model for WAAM will be built in this section. WAAM process is dominated by certain process parameters, such as welding speed and wire feed speed (WFS). In this study, WFS was selected as control input, and a series of Pseudo-Random Ternary Signal of WFS was utilized to stimulate the system continuously. The system input and output in experiments of system identification are presented as Figure 2.

According to previous studies [23], WAAM is a fuzzy and nonlinear process. Nowdays, artificial intelligence is more and more used to describe some complex process [24] [25]. In this study, the adaptive neuro fuzzy inference system (ANFIS) is utilized to model the WAAM process. ANIFS is a data-driven algorithm, which can model the dynamic process with human knowledge and reasoning processes [26]. ANFIS integrates both advantages of fuzzy inference and neural network. Most ANFIS algorithms are based on Sugeno-Type (TS) fuzzy model. Through training a neural network by system I/O data, the model structural parameters of TS fuzzy model could be identified. The TS fuzzy model has the forms as:

$R^{i}$ : If $x_{1}$ is $A_{i 1}$ and $x_{2}$ is $A_{i 2} \ldots$ and $x_{n}$ is $A_{i n}$, then $y_{i}=a_{0}+a_{1}^{i} x_{1}+\cdots+a_{n}^{i} x_{n}$.

Where:

- $x_{1} \ldots x_{n}$ are the input variables of ANFIS,

- $A_{i}$ and $B_{i}$ are the fuzzy sets,

- $y_{i}$ is the output of $i$ th rule

- $a_{0} \ldots a_{n}$ are the design parameters, which are determined by the neural network.

The final output of ANIFS is:

$$
y=\sum_{1}^{n} \overline{\omega_{i}} y_{i}
$$


Where $\overline{\omega_{i}}$ is the normalized firing strengths, which means the truth degree of each T-S rule. It can be determined by training the neural network. For more detail, please refer to relevant articles [26] [27].

In this study, previous control output y $(k-a), a=0,1$, and control input $u(k-b), b=0,1,2$, were selected as input of ANFIS, and the output of ANFIS was y $(\mathrm{k}+1)$. The modeling result of ANFIS is illustrated in Figure 3 (a). It can be observed that ANFIS model has high accuracy. For comparison, ARX model is also built (as shown in Figure 3 (b)). It can be seen that ANFIS has better predictive performance than ARX model. The mean square error (MSE) for the ARX model is 0.339, while this value for ANFIS is 0.103. There are several reasons responsible for the modelling error. Firstly, WAAM is a complex process and has uncertainty [28], which makes the model error inevitable. Secondly, due to the limited training data, the model could only fit the WAAM process to a certain extent, since the over-fitting or under-fitting may exist [29]. Besides, the fluctuation in equipment and welding process will generate disturbance, which is also responsible for the modelling error.
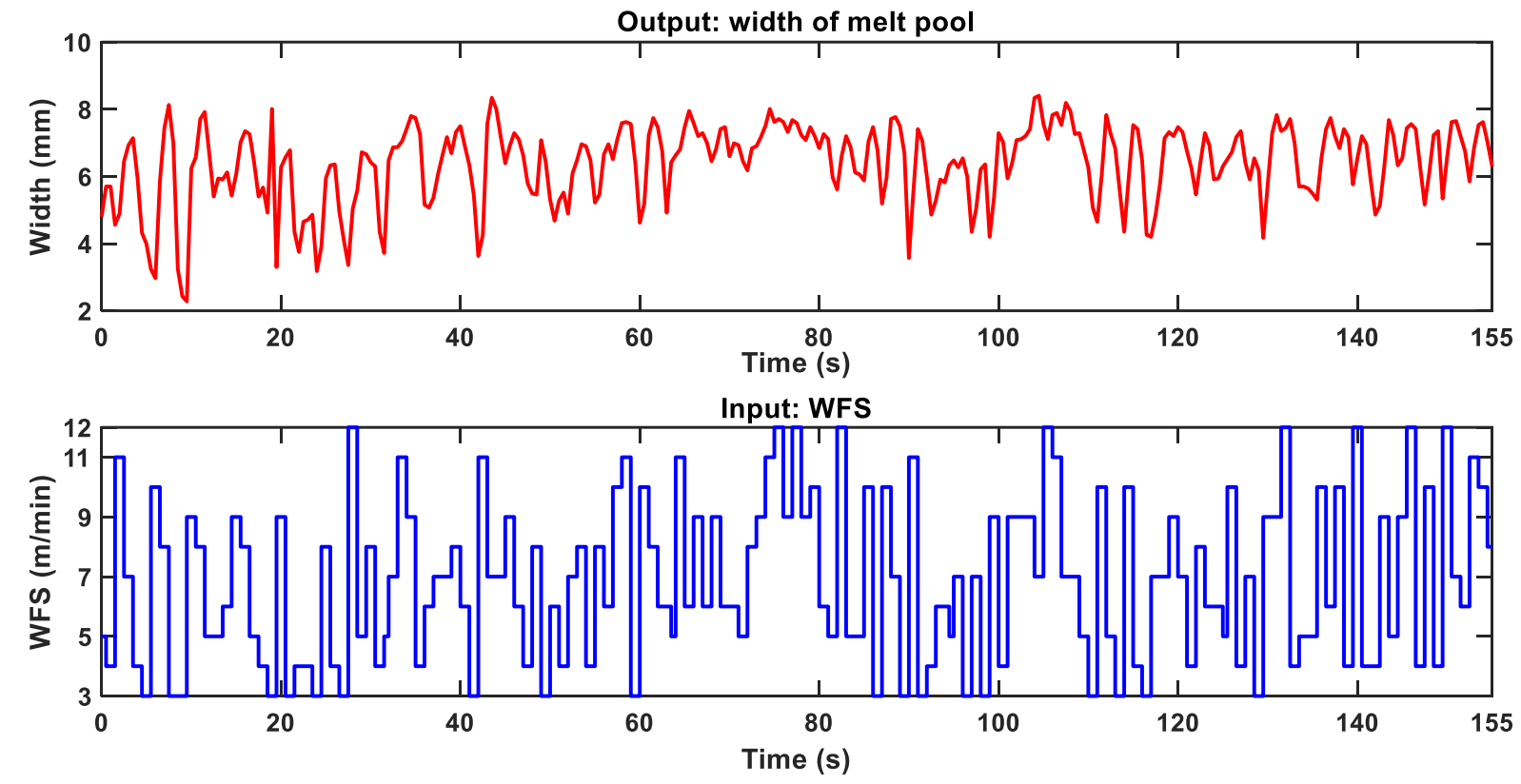

Figure 2 Response and Pseudo-Random Ternary Signal input 


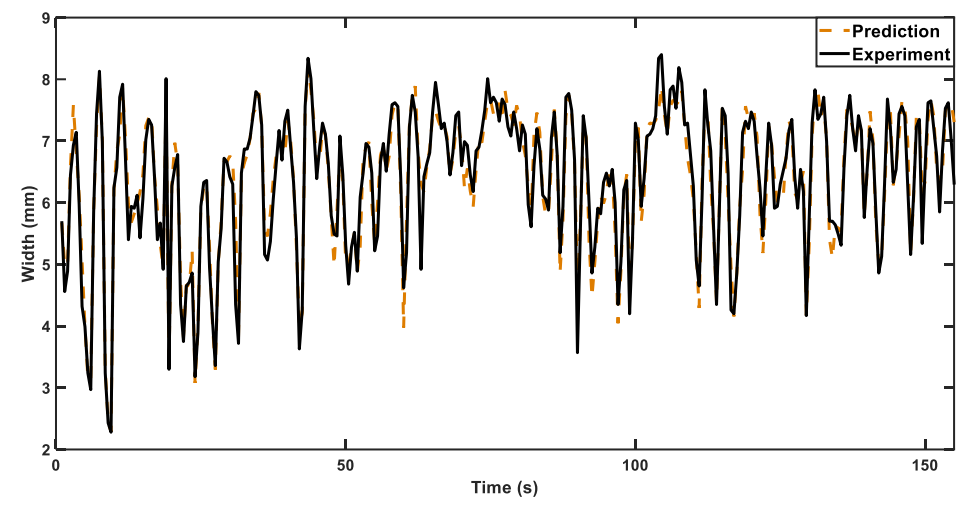

(a)

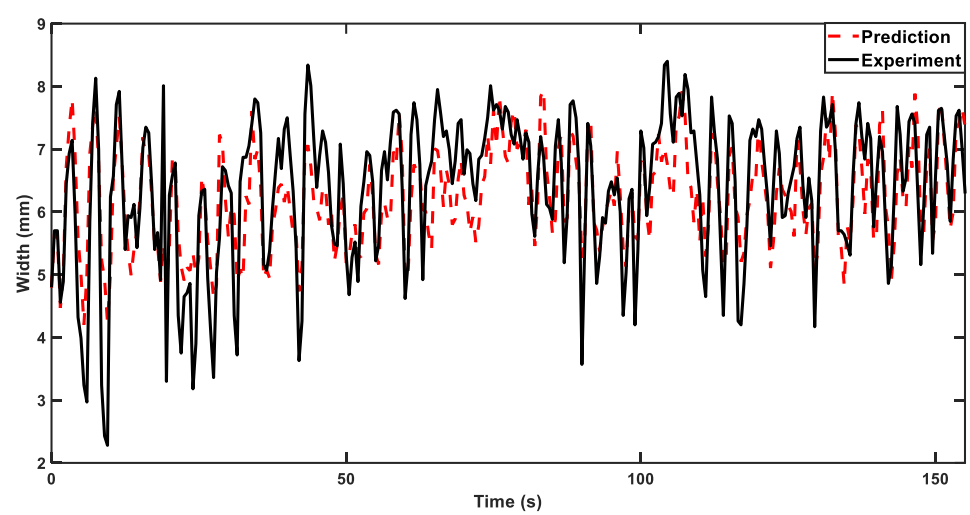

(b)

Figure 3 Prediction performance (a). ANFIS (b). ARX

Through the modelling process, it can be seen that building an accurate mathematical model for a practical plant is a hard task. Even though the model of the controlled plant is established, model uncertainty may exist. And in practice, the plant system may be time-varying, which will lead to a mismatch of model. From this point of view, an iterative learning control strategy based on online optimization by model-free adaptive algorithm needs to be developed for layer width control in WAAM.

The identified plant model will be only used for simulation, and controller design will not require any model information.

\section{MFALIC algorithm design}

\subsection{Iterative learning control}

Iterative learning control is a data-driven approach which aims to improve the tracking performance by introducing error in previous iterations for optimize control input in the next iteration. The basic algorithm for iterative learning control can be written as:

$$
u_{k+1}(t)=u_{k}(t)+L e_{k}(t)+\Gamma \dot{e}_{k}(t)+\Psi \int_{0}^{t} e_{k}(\tau) d \tau
$$

Equation (3) is the expression of PID-type ILC. For some other forms of ILC, like D-type, Ptype and PD-type, they can be formed by choosing various terms from the proportional, integral, 
and derivative terms. Besides, according to the error term, ILC can be divided into open loop and closed loop. When the error term only consider the error in previous iterations, the algorithm is open loop type. If the errors in current iterations are considered, the type of ILC algorithm is closed loop. In this study, open loop algorithm is selected.

\subsection{Model free adaptive iterative learning control}

Assuming the system model can be expressed as:

$$
y_{k}(t+1)=f\left(y_{k}(t), u_{k}(t)\right)
$$

Where $y_{k}(t), u_{k}(t)$ denote the control output and input. $\mathrm{K}$ represents the iteration index, and $\mathrm{t}$ is the time instance.

It's assumed that the system satisfied that:

Assumption 1. The partial derivative of $f(\cdot)$ with respect to control input $u_{k}(t)$ is continuous.

Assumption 2. The system satisfies the condition of generalized Lipschitz along the iteration axis:

$$
\left\|\Delta y_{k}(t+1)\right\| \leq b\left\|\Delta u_{k}(t)\right\|
$$

Where

$$
\begin{gathered}
\Delta y_{k}(t+1)=y_{k}(t+1)-y_{k-1}(t+1) \\
\Delta u_{k}(t)=u_{k}(t)-u_{k-1}(t)
\end{gathered}
$$

Assumption 1 is a general condition for controller design. It means that if the control input is continuous, then the system output will be continuous. Assumption 2 introduces constrain on the changing rate between output and input.

Based on above assumptions, the following theorems are introduced:

Theorem: If a nonlinear system satisfies assumption 1 and assumption 2 , if $\Delta u_{k}(t) \neq 0$, then there is an time-varying and iteration-dependent parameter $\phi_{k}(t)$, named pseudo-partialderivative (PPD), and $\left|\phi_{k}(t)\right|<=b$, such that the system could be described as compact form dynamic linearization (CFDL) data model (Proof in [30] [31]):

$$
\Delta y_{k}(t+1)=\phi_{k}(t) \Delta u_{k}(t)
$$

In order to find an appropriate input to achieve tacking desired output, a cost function is selected as:

$$
J\left(u_{k}(t)\right)=\left|e_{k}(t+1)\right|^{2}+\lambda\left|u_{k}(t)-u_{k-1}(t)\right|^{2}
$$

Where

$$
e_{k}(t+1)=y_{d}(t+1)-y_{k}(t+1)
$$

$\lambda$ is the weight coefficient. From equation (6) and (7), we obtain:

$$
J\left(u_{k}(t)\right)=\lambda\left|u_{k}(t)-u_{k-1}(t)\right|^{2}+\left|e_{k-1}(t+1)-\phi_{k}(t)\left[u_{k}(t)-u_{k-1}(t)\right]\right|^{2}
$$

Let $\frac{\partial J\left(u_{k}(t)\right)}{\partial u_{k}(t)}=0$, and the control law can be obtained as: 


$$
u_{k}(t)=u_{k-1}(t)+\frac{\rho \phi_{k}(t)}{\lambda+\left|\phi_{k}(t)\right|^{2}} e_{k-1}(t+1)
$$

Where $\rho$ is the step factor, and $\lambda$ is a weight factor. $\phi_{k}(t)$ is unknown, and need to be estimated. We use $\hat{\phi}_{k}(t)$ to present the estimated $\phi_{k}(t)$, and the cost function for estimating $\phi_{k}(t)$ can be selected as:

$$
J\left(\hat{\phi}_{k}(t)\right)=\mu\left|\hat{\phi}_{k}(t)-\hat{\phi}_{k-1}(t)\right|^{2}+\left|\Delta y_{k-1}(t+1)-\hat{\phi}_{k}(t) \Delta u_{k-1}(t)\right|^{2}
$$

Where $\mu$ is a positive weight coefficient. Through setting $\frac{\partial J\left(\widehat{\phi}_{k}(t)\right)}{\partial u_{k}(t)}=0, \widehat{\phi}_{k}(t)$ can be calculated as:

$$
\hat{\phi}_{k}(t)=\hat{\phi}_{k-1}(t)+\frac{\eta \Delta u_{k-1}(t)}{\mu+\left|\Delta u_{k-1}(t)\right|^{2}}\left[\Delta y_{k-1}(t+1)-\hat{\phi}_{k-1}(t) \Delta u_{k-1}(t)\right]
$$

Where $\eta$ is the step length factor. To enhance the tracking ability of algorithm, a reset algorithm for $\hat{\phi}_{k}(t)$ is designed as:

$$
\left.\hat{\phi}_{k}(t)=\hat{\phi}_{k}(0) \quad \text { if } \hat{\phi}_{k}(t) \leq \varepsilon \text { or }\left|\Delta u_{k}(t)\right| \leq \varepsilon\right)
$$

Where $\varepsilon$ is a small positive value, and $\widehat{\phi}_{k}(0)$ is the initial value in each iteration.

From the learning law (equation (9)), it can be seen that it's similar with the conventional Ptype iterative learning control. However, its learning parameters are updated through online I/O data calculations. Therefore, MFAILC combines the advantages of both online learning and adaption from ILC and Model Free Adaptive Control. This process can be described by Figure 4.

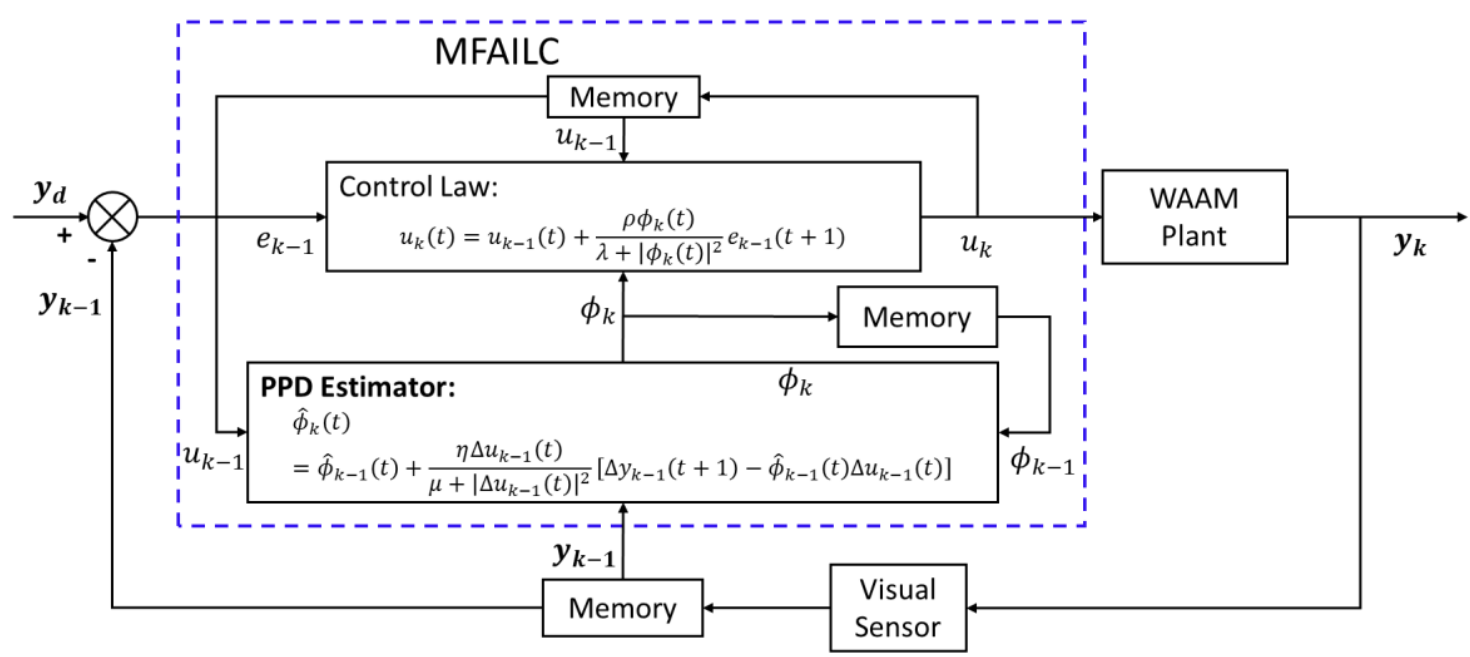

Figure 4 The structure of MFAILC

Based on above derivation, the MFAILC could be concluded as:

$$
\begin{gathered}
\hat{\phi}_{k}(t)=\hat{\phi}_{k-1}(t)+\frac{\eta \Delta u_{k-1}(t)}{\mu+\left|\Delta u_{k-1}(t)\right|^{2}}\left[\Delta y_{k-1}(t+1)-\hat{\phi}_{k-1}(t) \Delta u_{k-1}(t)\right] \\
\hat{\phi}_{k}(t)=\hat{\phi}_{k}(0)\left(\text { if } \hat{\phi}_{k}(t) \leq \varepsilon \text { or }\left|\Delta u_{k}(t)\right| \leq \varepsilon\right) \\
u_{k}(t)=u_{k-1}(t)+\frac{\rho \phi_{k}(t)}{\lambda+\left|\phi_{k}(t)\right|^{2}} e_{k-1}(t+1)
\end{gathered}
$$

The analysis of the stability and convergence of MFAILC can be found in reference [27] [32] 


\section{Simulation}

In order to investigate the effectiveness of MFAILC for WAAM control, simulation is conducted using the ANFIS plant model, which is described in the section 2. The ANFIS model only provide I/O data for simulation. There isn't any information about the dynamic model will be utilized in MFAILC scheme.

The desired value of layer width is defined as:

$$
y_{d}=0.3 \sin (k \pi / 50)+0.4 \cos (k \pi / 100)
$$

The parameters of controller were selected as: $\eta=1, \rho=1.5, \mu=\lambda=0.5, \varepsilon=10^{-4}$. The initial condition of the simulation is set as: $u_{0}(t)=0, u_{1}(t)=0$. The simulation result is presented in Figure 5. It can be observed that the tracking error is continuously reduced through learning iteratively and pole level of tracking error can be achieved at 7 th iterations.

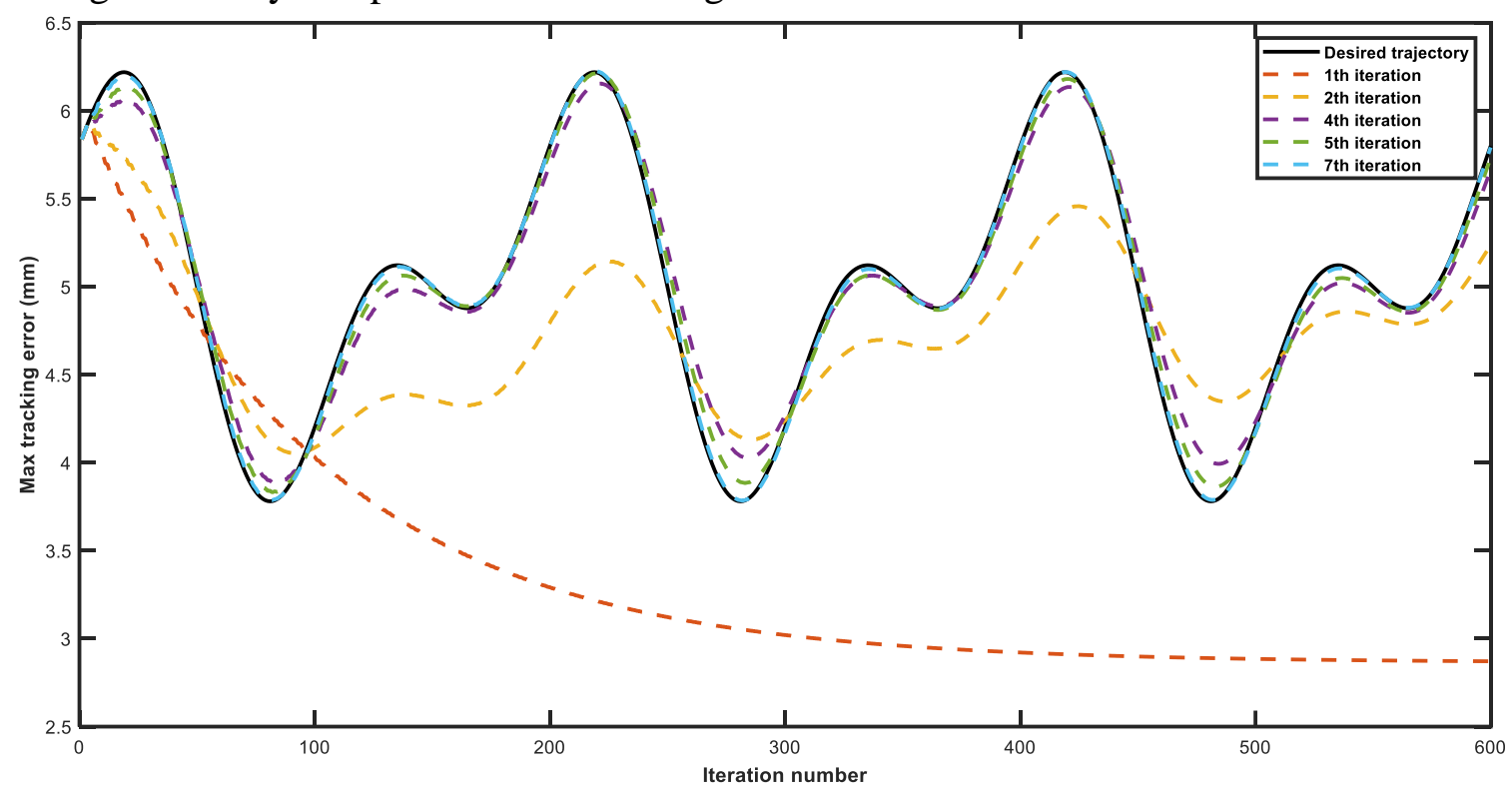

Figure 5 Trajectory tracking results of MFAILC

For comparison, a conventional PID (proportional-integral-derivative) controller was utilized for simulation of control. When $K_{p}=0.5, K_{i}=-0.2, K_{d}=0.1$, the best control performance can be achieved. The tracking performance is presented in Figure 6. It can be seen that significant control delay was generated by PID when tracking time-varying desired value, while proposed MFAILC could compensate the time delay based on last iteration experience. This is because PID has limited responding speed and the tracking trajectory is changing rapidly. Compared to MFAICL, the lack of priori knowledge in PID controller also make it hard to tacking changing trajectory. Also, the maximum tracking error in each iteration controlled by MFAICL and original P-type ILC was compared, as shown is Figure 7. It can be observed that the MFAILC algorithm has faster converging speed and better tracking performance than P-type ILC.

The simulation results demonstrate the superiority of proposed MFAICL in controlling repetitive WAAM process. Besides, the parameters tuning of ILC or PID controller is timeconsuming, since a number of experiments need to be done offline to ensure a set of appropriate parameters, while MFAILC algorithm could adjust its parameters adaptively. Thus, it can be concluded that MFAILC algorithm has both superior control performance and better applicability. 


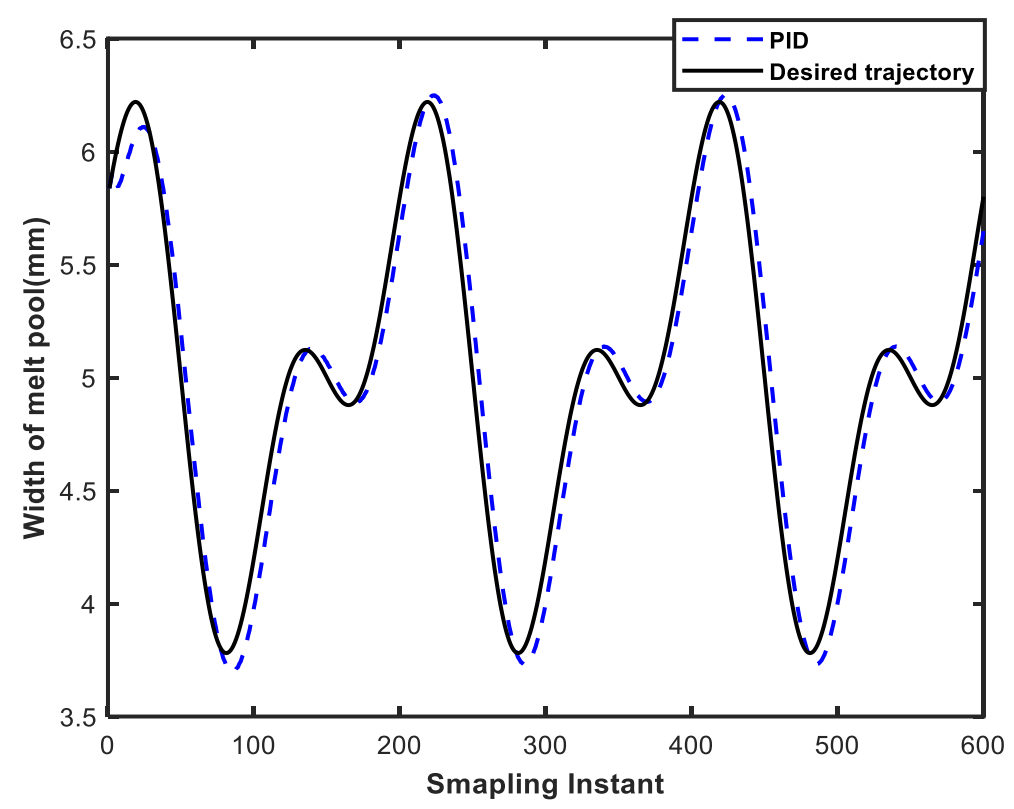

Figure 6 Tracking performance of PID algorithm

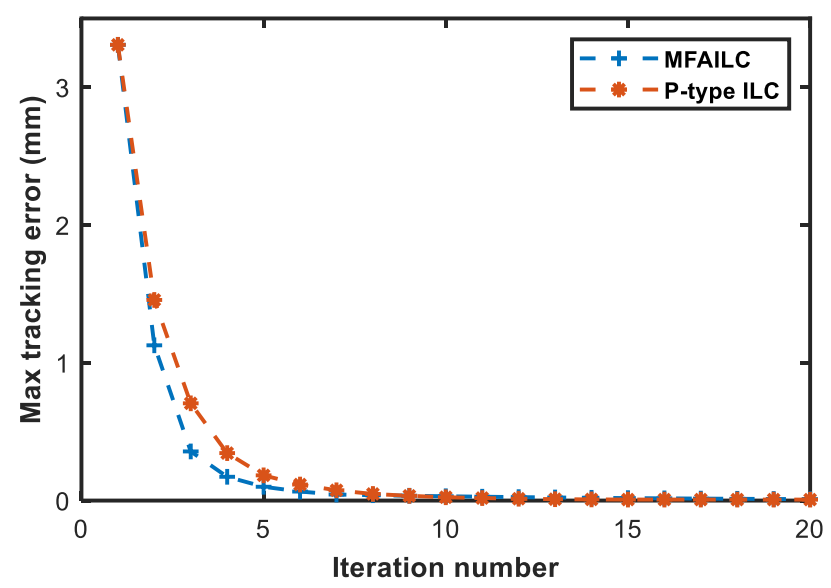

Figure 7 Comparison of maximum tracking error 


\section{Experiment}

\subsection{Setup}

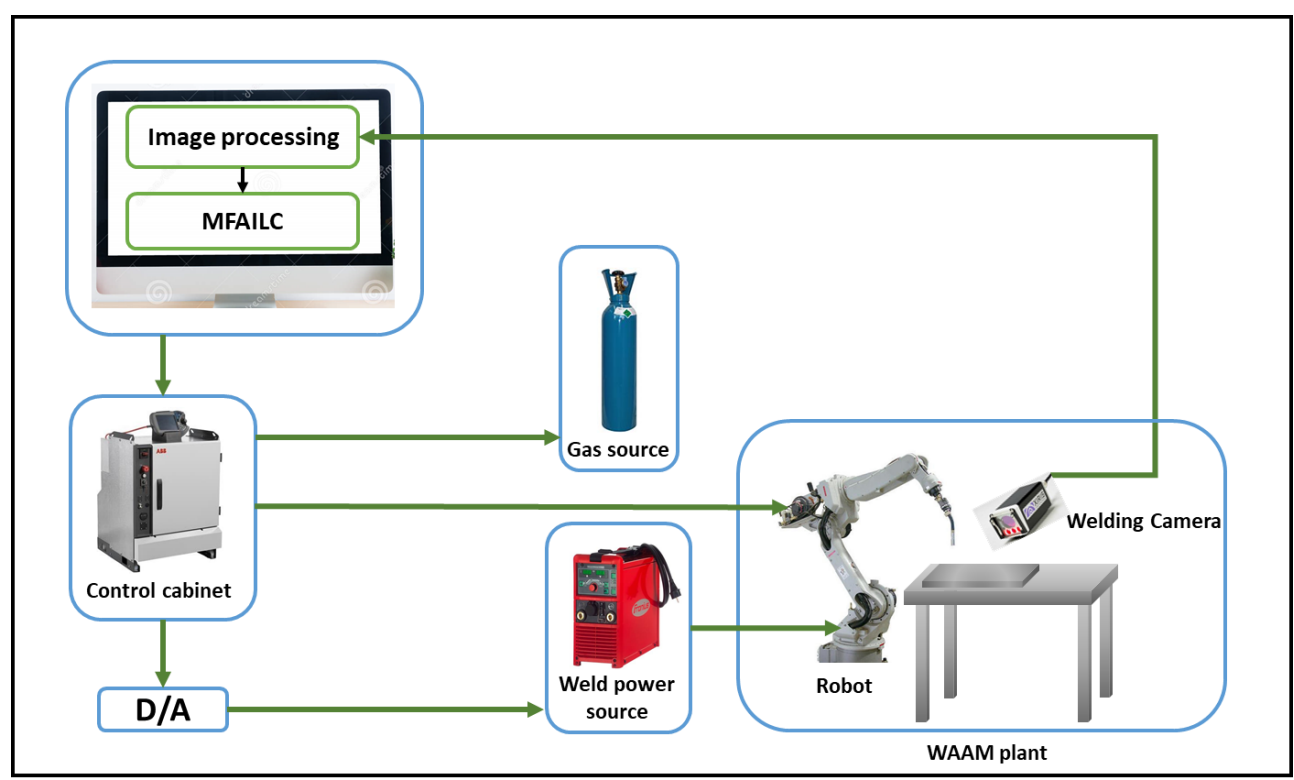

Figure 8 Experimental system

The experiment system is presented Figure 8. It consists of central computer, Fronius CMT welder, protective gas source, Xiris XVC-1000E welding camera, ABB robot and robot controller. In welding system, the adjustable parameters is the WFS (wire feed speed), and other parameters (welding voltage and current) will automatically match the setting of WFS. The shielding gas consists of $80 \% \mathrm{Ar}$ and $20 \% \mathrm{CO}_{2}$ with a flow rate of $25 \mathrm{~L} / \mathrm{min}$. A $1.2 \mathrm{~mm}$ diameter of steel ER70S-6 filler wire was utilized. An optical filter with $650 \mathrm{~nm}$ central wavelength is combined with welding camera to constrain the strong CMT welding arc.

The images of melt pool are obtained by Xiris XVC-1000E welding camera. As shown in Figure 9, a control program was developed to implement image acquisition and processing, calculation of control input and hardware communication. As illustrated in Figure 10, through obtaining the Region of Interest (ROI) in welding image, and extracting the edge of melt pool, the width of melt pool can be measured. A simple calibration of the camera was implemented. The relationship between the dimension in central area of image and in real world is obtained. The actual width of single pixel is about $0.03 \mathrm{~mm} /$ pixel. Therefore, the actual width can be calculated as the following equation:

$$
\text { Width }_{\text {actual }}=0.03 \text { Width }_{\text {img }}
$$




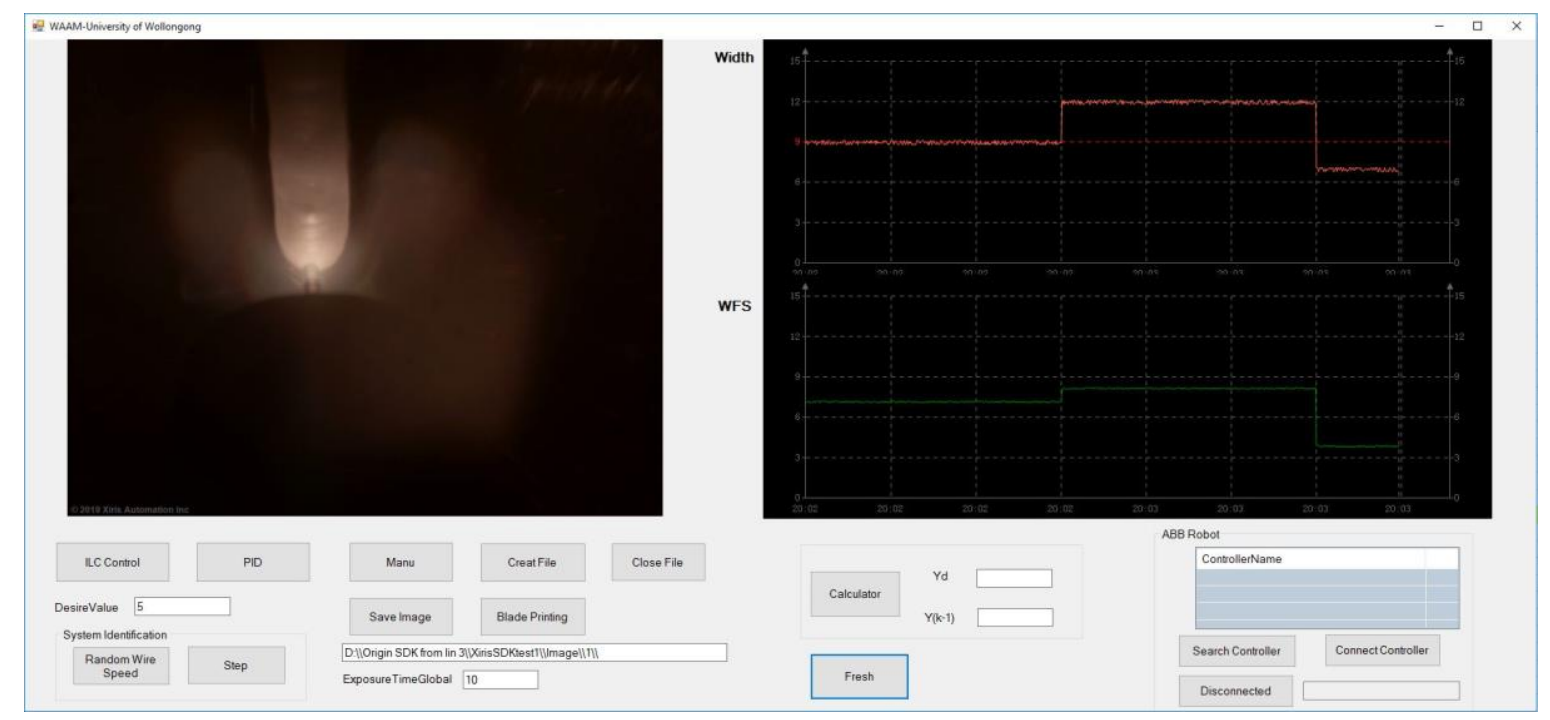

Figure 9 Interface of MFAICL control program

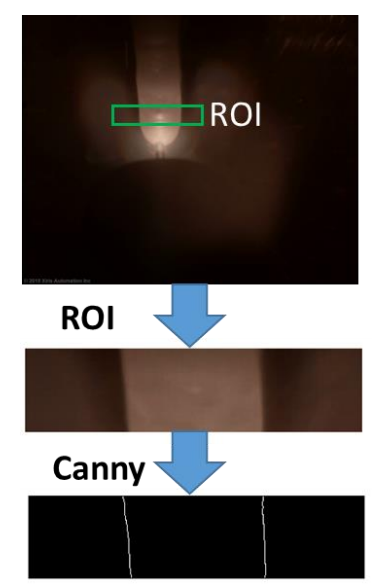

Figure 10 Flowchart of edge extracting

Table 1 Parameter of XIRIS welding camera

\begin{tabular}{ccccc}
\hline Exposure time & AGC latency & Video average & Saturation & Gamma \\
\hline $25 \mathrm{~ms}$ & $75 \%$ & 4 & $40 \%$ & $50 \%$ \\
\hline
\end{tabular}

\subsection{Experiment result}

\subsubsection{Tracking performance}

To further prove the effectiveness of the MFAILC algorithm in controlling the width of melt pool during WAAM process, the experiments were conducted. Firstly, the controller was designed to track step changing set point to investigate its tacking ability. To present the response process of iterative learning, a lower initial control input (WFS=3 $\mathrm{m} / \mathrm{min}$ ) for the first bead is selected. As shown in Figure 11, the desired trajectory was set to be $7 \mathrm{~mm}$ at initial period, and change to $12 \mathrm{~mm}$ and $9 \mathrm{~mm}$ over time. The parameters of MFAILC controller were set as: $\eta=1, \rho=1.5, \mu=\lambda=0.5, \varepsilon=10^{-4}$. In WAAM, depositing one bead represents one 
iteration. From Figure 11, it can be seen that through learning iteratively bead by bead, MFAILC algorithm is capable to track desired trajectory accurately at 3th iteration. This illustrates that the MFALIC algorithm has good tracking ability and fast responding speed in the control of WAAM process. The fluctuation of the melt pool width in these experiments is less than $0.2 \mathrm{~mm}$, which is considered acceptable in our application. Figure 12 presents the real appearance of the bead deposited under control of MFAILC.
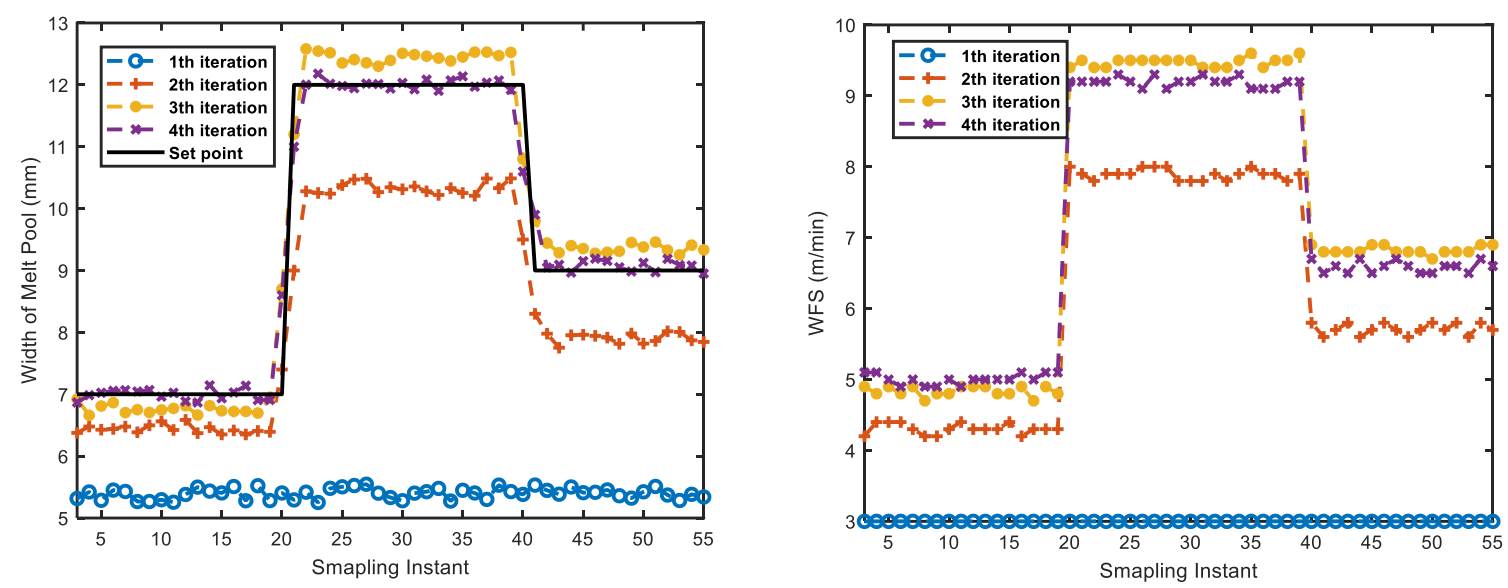

Figure 11 Tracking performance

(a)

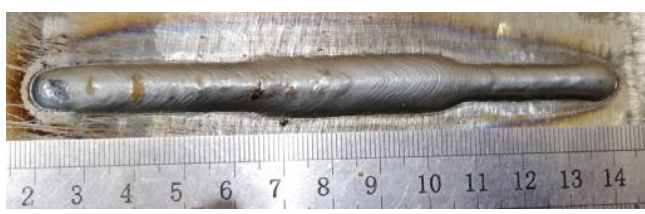

(b)

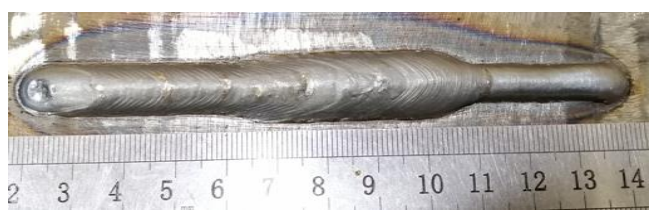

(c)

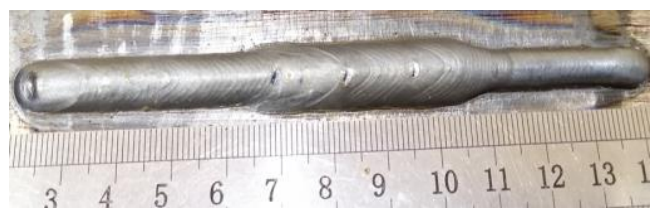

Figure 12 Appearances of weld beads in tracking experiments (a). 2th iteration (b). 3th iteration (c). 4th iteration

\subsubsection{Robust performance}

\section{A. Disturbance of welding speed}

In WAAM, the welding speed is a major factor which has great impact on melt pool width. Therefore, a step change of the travelling speed was employed to examine the robustness of proposed controller. During the first iteration in experiment, a step change of welding speed 
was introduced, which change to $6 \mathrm{~mm} / \mathrm{s}$ from $3 \mathrm{~mm} / \mathrm{s}$, and then restore to $3 \mathrm{~mm} / \mathrm{s}$. As can be seen in Figure 13, the melt pool width decreased immediately due to the step change in the speed. During the following iterations, MFAILC is triggered and the controller is able to adjust the WFS according to the measured melt pool width at next sampling instant in the last iterations. It can be seen that through online iterative learning, the melt pool width can revert to desired set-point under the control of MAFILC, despite the disturbance of step change in welding speed. Figure 14 presents the appearances of weld beads in robustness experiments.

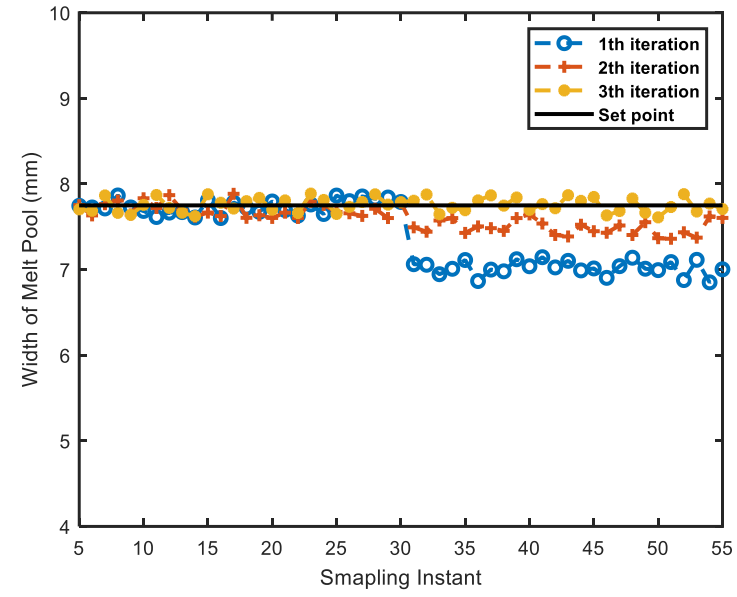

(a)

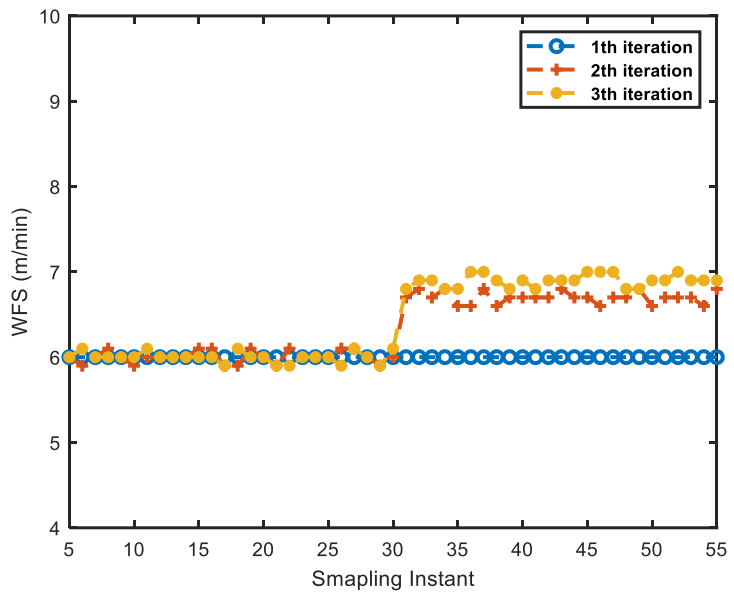

(b)

Figure 13 Results of control experiment under welding disturbance. (a) Melt pool width. (b) Control input

(a)

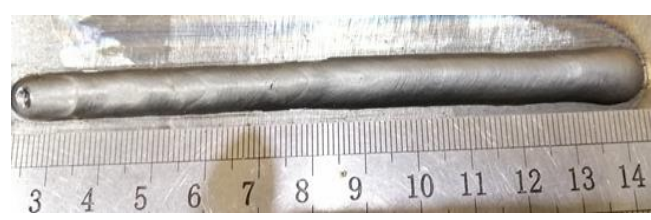

(b)

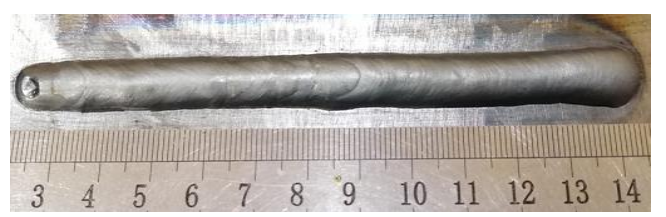

(c)

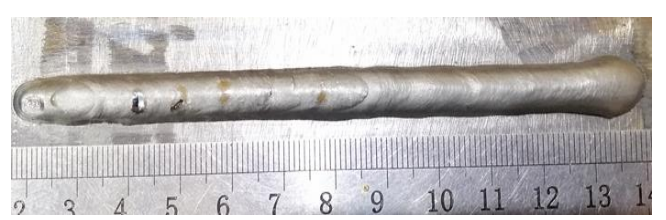

Figure 14 Appearances of weld beads in robustness experiments (a). 1h iteration (b). 2th iteration (c). 3th iteration

\section{B. Disturbance of stick-out length}

The length of stick out is another factor, which has effect on the width of melt pool during WAAM process. In this experiment, the length of stick out was set to be a disturbance variables. During deposition, the stick-out length was $5 \mathrm{~mm}$ at initial period, and it was increased to $20 \mathrm{~mm}$ at 30th sampling instant. From Figure 15, it can be seen that the width of melt pool increased due to the disturbance in stick-out length. In the second iterations, the MAILC controller was 
triggered, and the WFS was adjusted to compensate the change of melt pool width caused by the stick out length of wire. It can be observed that the disturbance of stick-out length can be overcome through 2-3 iterations. The disturbance experiments demonstrate the robustness of MFAILC algorithm in controlling the width of melt pool during WAAM process.

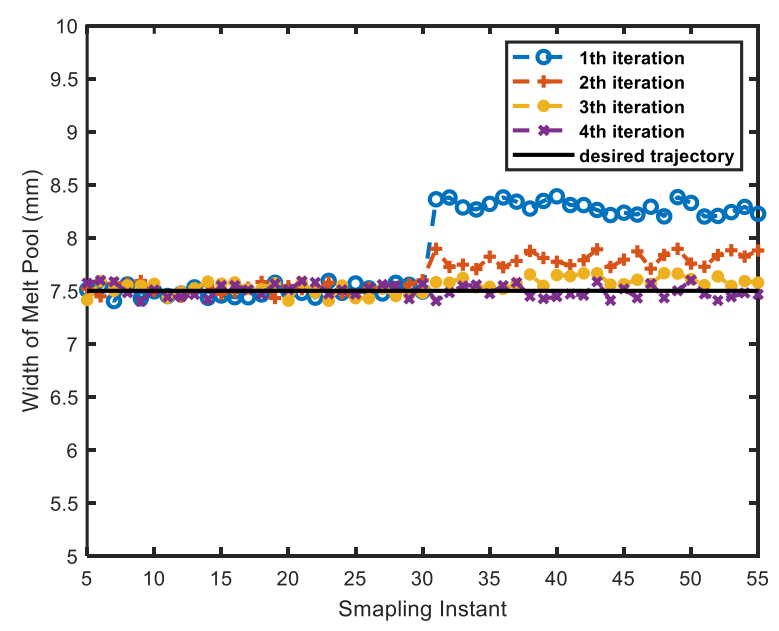

(a)

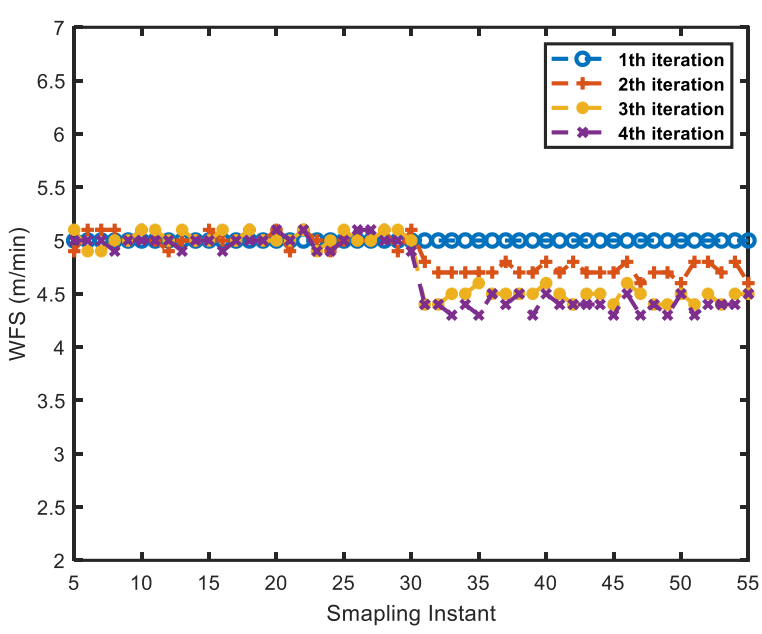

(b)

Figure 15 Results of control experiment under stick-out length disturbance. (a) Melt pool width. (b) Control input

Figure 16 presents a wall of variable width, which is deposited under the control of MFAILC. From the experiments results, we can conclude that the proposed MFAILC can control the width of melt pool effectively. The value of the melt pool width can converge to desired trajectory under initial value shifting condition under the control of MFAILC. Also, MFAICL can effectively resist external disturbances during iterations. Additionally, the design of MFAILC controller only require the data of input and output, which omit the complicated process of dynamic modelling.

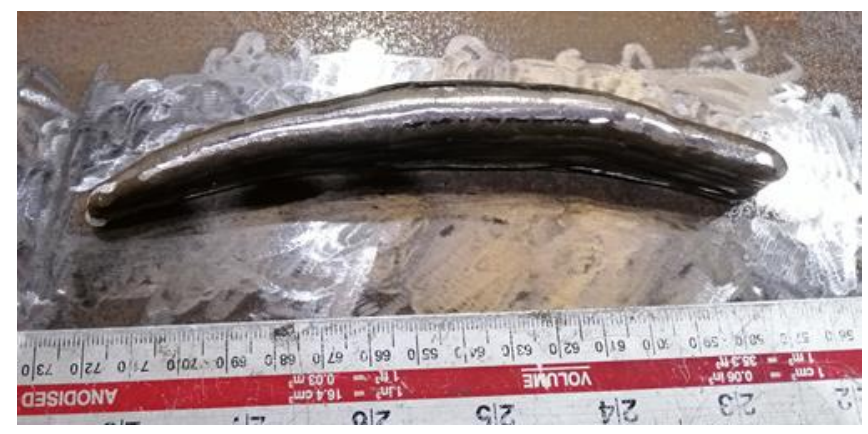

Figure 16 Wall of variable width deposited by MFAILC controller

\section{Conclusion}

In this paper, a MFAILC-based width of melt pool control strategy was proposed for WAAM process. Firstly, an innovative passive visual sensing system was designed to measure the realtime width of melt pool. Then dynamic experiments were conducted and an adaptive nonlinear 
ANFIS algorithm was used to model the dynamic process of WAAM. In validation phase, a value of 0.103 for MSE could be achieved by ANFIS in the prediction. Furthermore, a discrete time MFAILC algorithm was derived. The design of this controller only based on the I/O data in WAAM process without demand any priori information of the control system. Based on ANFIS model, simulation is performed. The simulation results demonstrate the effectiveness of the MFAILC controller, as well as its superior control performance to PID and P-ILC. The MFAILCA could achieve pole error tracking after 6 iterations while there is obvious static error in PID control. Compared to normal P-ILC controller, the MFAILC has shorter response iterations. The ANFSI model was only used as simulation plant, no any model information was involved in controller design. To further validate the effectiveness of proposed algorithm, the tracking performance and robustness of this MFAILC controller are verified by experiments. In tracking experiment, a good tracking performance can be achieved after 3 iterations. In robustness experiment, different disturbances can be eliminated within 3 iterations. The experiment results validate the good performance of MFAILC in tracking and anti-interference.

In the future, the geometry of melt pool will be controlled considering the situation of overlap of different beads. Besides, the height of the deposited bead will be regulated by advanced control algorithms.

\section{Acknowledgements}

The authors gratefully acknowledge the China Scholarship Council for financial support (NO. 201704910782) and UOW Welding and Industrial Automation Research Centre. 


\section{References}

1. Guo N, Leu MC (2013) Additive manufacturing: technology, applications and research needs. Frontiers of Mechanical Engineering 8 (3):215-243. doi:10.1007/s11465-013-0248-8

2. Thomas CL, Gaffney TM, Kaza S, Lee CH Rapid prototyping of large scale aerospace structures. In: 1996 IEEE Aerospace Applications Conference. Proceedings, 1998. IEEE, pp 219-230

3. Song Y, Yan Y, Zhang R, Xu D, Wang F (2002) Manufacture of the die of an automobile deck part based on rapid prototyping and rapid tooling technology. Journal of materials processing technology $120(1-3): 237-242$

4. Giannatsis J, Dedoussis V (2009) Additive fabrication technologies applied to medicine and health care: a review. The International Journal of Advanced Manufacturing Technology 40 (1-2):116-127

5. Sachlos E, Czernuszka J (2003) Making tissue engineering scaffolds work. Review: the application of solid freeform fabrication technology to the production of tissue engineering scaffolds. Eur Cell Mater 5 (29):39-40

6. Pham DT, Dimov SS (2003) Rapid prototyping and rapid tooling-the key enablers for rapid manufacturing. Proceedings of the Institution of Mechanical Engineers, Part C: Journal of Mechanical Engineering Science 217 (1):1-23

7. Kianian B (2016) Wohlers Report 2016: 3D Printing and Additive Manufacturing State of the Industry, Annual Worldwide Progress Report: Chapter title: The Middle East.

8. Williams SW, Martina F, Addison AC, Ding J, Pardal G, Colegrove P (2016) Wire+ arc additive manufacturing. Materials Science and Technology 32 (7):641-647

9. Tapia G, Elwany A (2014) A review on process monitoring and control in metal-based additive manufacturing. Journal of Manufacturing Science and Engineering 136 (6):060801

10. Xu F, Dhokia V, Colegrove P, McAndrew A, Williams S, Henstridge A, Newman ST (2018) Realisation of a multi-sensor framework for process monitoring of the wire arc additive manufacturing in producing Ti-6Al-4V parts. International Journal of Computer Integrated Manufacturing 31 (8):785-798. doi:10.1080/0951192x.2018.1466395

11. Yu Kang L, Yu Ming Z (2014) Model-Based Predictive Control of Weld Penetration in Gas Tungsten Arc Welding. IEEE Transactions on Control Systems Technology 22 (3):955-966. doi:10.1109/tcst.2013.2266662

12. Liu Y, Zhang Y (2013) Control of 3D weld pool surface. Control Engineering Practice 21 (11):14691480. doi:10.1016/j.conengprac.2013.06.019

13. Liu Y, Zhang W, Zhang Y (2015) Dynamic Neuro-Fuzzy-Based Human Intelligence Modeling and Control in GTAW. IEEE Transactions on Automation Science and Engineering 12 (1):324-335. doi:10.1109/tase.2013.2279157

14. Xiong J, Zou S (2019) Active vision sensing and feedback control of back penetration for thin sheet aluminum alloy in pulsed MIG suspension welding. Journal of Process Control 77:89-96. doi:10.1016/j.jprocont.2019.03.013

15. Doumanidis C, Kwak Y-M (2002) Multivariable adaptive control of the bead profile geometry in gas metal arc welding with thermal scanning. International Journal of Pressure Vessels and Piping 79 (4):251-262. doi:10.1016/S0308-0161(02)00024-8

16. Doumanidis C, Kwak Y-M (2001) Geometry modeling and control by infrared and laser sensing in thermal manufacturing with material deposition. Journal of manufacturing science and engineering 123 (1):45-52. doi:10.1115/1.1344898

17. Xiong J, Yin Z, Zhang W (2016) Closed-loop control of variable layer width for thin-walled parts in wire and arc additive manufacturing. Journal of Materials Processing Technology 233:100-106

18. Xiong J, Zhang G (2014) Adaptive control of deposited height in GMAW-based layer additive manufacturing. Journal of Materials Processing Technology 214 (4):962-968. doi:10.1016/j.jmatprotec.2013.11.014

19. Bu X, Wang S, Hou Z, Liu W (2019) Model free adaptive iterative learning control for a class of nonlinear systems with randomly varying iteration lengths. Journal of the Franklin Institute 356 (5):24912504. doi:10.1016/j.jranklin.2019.01.003

20. Hou Z, Chi R, Gao H (2016) An overview of dynamic-linearization-based data-driven control and applications. IEEE Transactions on Industrial Electronics 64 (5):4076-4090

21. Hou Z, Jin S (2011) Data-driven model-free adaptive control for a class of MIMO nonlinear discretetime systems. IEEE Transactions on Neural Networks 22 (12):2173-2188

22. Chi R, Hou Z, Xu J (2008) Adaptive ILC for a class of discrete-time systems with iteration-varying trajectory and random initial condition. Automatica 44 (8):2207-2213 
23. Huang N, Liu Y, Chen S, Zhang $Y$ (2015) Interval model control of human welder's movement in machine-assisted manual GTAW torch operation. The International Journal of Advanced Manufacturing Technology 86 (1-4):397-405. doi:10.1007/s00170-015-8153-4

24. Faizabadi MJ, Khalaj G, Pouraliakbar H, Jandaghi MR (2014) Predictions of toughness and hardness by using chemical composition and tensile properties in microalloyed line pipe steels. Neural Computing and Applications 25 (7-8):1993-1999

25. Pouraliakbar H, Khalaj M-j, Nazerfakhari M, Khalaj G (2015) Artificial neural networks for hardness prediction of HAZ with chemical composition and tensile test of X70 pipeline steels. Journal of Iron and Steel Research International 22 (5):446-450

26. Pouraliakbar H, Nazari A, Fataei P, Livary AK, Jandaghi M (2013) Predicting Charpy impact energy of Al6061/SiCp laminated nanocomposites in crack divider and crack arrester forms. Ceramics International 39 (6):6099-6106

27. Alarifi IM, Nguyen HM, Naderi Bakhtiyari A, Asadi A (2019) Feasibility of ANFIS-PSO and ANFISGA Models in Predicting Thermophysical Properties of Al2O3-MWCNT/Oil Hybrid Nanofluid. Materials (Basel) 12 (21). doi:10.3390/ma12213628

28. Santos T, Caetano R, Lemos JM, Coito FJ (2000) Multipredictive adaptive control of arc welding trailing centerline temperature. IEEE Transactions on Control systems technology 8 (1):159-169

29. Pouraliakbar H, Firooz S, Jandaghi MR, Khalaj G, Nazari A (2016) Predicting the ultimate grain size of aluminum sheets undergone constrained groove pressing. The International Journal of Advanced Manufacturing Technology 86 (5-8):1639-1658

30. Alfaro SC, Franco FD (2010) Exploring infrared sensoring for real time welding defects monitoring in GTAW. Sensors (Basel) 10 (6):5962-5974. doi:10.3390/s100605962

31. Bu X, Hou Z, Chi R (2013) Model free adaptive iterative learning control for farm vehicle path tracking. IFAC Proceedings Volumes 46 (20):153-158

32. Abu-Mahfouz I, El Ariss O, Esfakur Rahman AHM, Banerjee A (2017) Surface roughness prediction as a classification problem using support vector machine. The International Journal of Advanced Manufacturing Technology 92 (1-4):803-815. doi:10.1007/s00170-017-0165-9 